\title{
La articulación entre el Ministerio de Relaciones Exteriores y Culto y las Provincias argentinas, en un contexto de federalización de la Cooperación Descentralizada.*
}

por Pamela Curvale $e^{\star *}$

\section{- Resumen}

Las transformaciones internacionales de las últimas décadas han propiciado el aumento de la gestión externa de los actores subestatales, que consideran al escenario internacional como un espacio favorable para la internacionalización. El propósito de este artículo es realizar una descripción de las acciones y programas de Cooperación Descentralizada del Ministerio de Relaciones Exteriores y Culto, en su articulación con las provincias de Argentina en un marco de federalización.

\section{- Palabras Clave}

Cooperación Descentralizada - Federalismo - actores subestatales - gestión eterna - Ministerio de Relaciones Exteriores y Culto de la Nación - Provincias argentinas.

\section{Inglés | English}

\section{- Abstract}

The international transformations of the recent decades have led to the increase of the external management of sub-state agents, who consider the international scenario as a favorable space for internationalization. This article aims to describe the actions and programs of Decentralized Cooperation of the Ministry of Foreign Affairs and Worship in its articulation with the provinces of Argentina within federal setting.

\section{- Key Words}

Decentralized Cooperation - Federalism - sub-state actors external management - Ministry of Foreign Affairs and Worship of the Nation - Argentine Provinces.

\section{Introducción}

A partir de 1970, las unidades subestatales se incorporan a los estudios internacionales dando cuenta del surgimiento de nuevos actores, renovados temas y corrientes teóricas alternativas que irrumpen en el escenario global fomentando acciones de gestión externa como la Cooperación Descentralizada $(\mathrm{CD})$.

Por lo tanto, en la medida en que el escenario internacional fue cambiando y surgieron nuevos actores con la capacidad, habilidad y autonomía para actuar en el escenario internacional (Barbé, 2010), el paradigma estatocéntrico fue incapaz de explicar estas transformaciones, y fue cuestionado provocando la necesidad de desarrollar modelos alternativos en la disciplina de las Relaciones Internacionales. Por esta razón, surge el paradigma globalista que reconoce la existencia de múltiples actores internacionales y el surgimiento de nuevos temas globales, a partir de la afirmación de la interdependencia (Keohane y Nye, 1988).

En este contexto, el desarrollo de acciones de gestión externa, la Cooperación Descentralizada, el fortalecimiento del Estado federal y la vinculación entre los diferentes niveles gubernamentales, forman parte del proceso de cambio en el cual se destaca el rol de los actores subestatales.

El presente artículo tiene como objetivo describir la articulación entre el Ministerio de Relaciones Exteriores y Culto y las Provincias de Argentina, en un marco de federalización de la Cooperación Descentralizada.

Esta investigación fue realizada a partir de una metodología cualitativa, con un diseño exploratorio - descriptivo. Para lograr el objetivo propuesto se consideró como parte del corpus de análisis a los programas, informes y las acciones de difusión desarrolladas por la Dirección General de Cooperación Internacional y la Dirección de Asuntos Federales y Electorales, organismos pertenecientes al Ministerio de Relaciones Exteriores y Culto de la Nación.

\footnotetext{
* El siguiente trabajo fue realizado retomando ideas plasmadas en la Tesis de Maestría: "La articulación entre el Ministerio de Relaciones Exteriores y Culto y las Provincias argentinas, en un contexto de federalización de la Cooperación Descentralizada. El caso de la Provincia de Entre Ríos (2007-2015)", realizada por la autora en Julio de 2018, Maestría en Integración y Cooperación Internacional, Centro de Estudios Interdisciplinarios, Universidad Nacional de Rosario.
}

** Licenciada en Relaciones Internacionales, Licenciada en Ciencias Políticas - Universidad Católica Argentina, Magister en Integración y Cooperación Internacional - Universidad Nacional de Rosario. Contacto: pamelacurvale@uca.edu.ar 
Para llevar a cabo este estudio se emplearon las siguientes fuentes:

a. Entrevistas semiestructuradas: las mismas fueron realizadas a informantes claves, funcionarios públicos que, dentro de las áreas específicas del Ministerio de Relaciones Exteriores y Culto, juegan un rol destacado en la gestión y difusión de la Cooperación Internacional. Se entrevistó a la Directora del Programa Federal de Cooperación, Sra. Alicia San Nicolás, dependiente de la Dirección General de Cooperación Internacional y a la Directora de la Dirección de Asuntos Federales y Electorales, Mtro. María Cristina Tosonotti.

b. Material documental: análisis de 1. Legislaciones tales como la Constitución Nacional de Argentina (reformada en el año 1994) y los Decretos que versan sobre las funciones de los órganos especializados en Cooperación Descentralizada de Cancillería; 2. Notas de prensa e información digital: abarca los programas, proyectos, foros, encuentros y convenios implementados y difundidos por Cancillería en materia de Cooperación; y 3. Publicaciones: comprende las ediciones ya sea en formato papel o digital. La forma de recolección consistió en la solicitud de la información a las áreas competentes en la materia indagando en los archivos y la búsqueda en el portal público digital del Ministerio en el siguiente sitio: http://cooperacionarg.gob.ar/es/institucional. Criterios de búsqueda: "Cooperación Internacional", "Cooperación Descentralizada", "Programas de Cooperación con las provincias" y "Federalización de la cooperación".

La presente investigación fue abordada desde la perspectiva del paradigma globalista o transnacionalista y análisis del escenario internacional desde la interdependencia compleja, retomando conceptos centrales tales como Cooperación Descentralizada; Federalismo; gestión externa y actores subestatales o actores gubernamentales no centrales.

Richard W. Mansbach, Yale H. Ferguson y Donald E. Lampert, autores transnacionalistas representantes del llamado "paradigma del sistema global" elaboraron una nueva tipología de actores internacionales que engloba a los tradicionales, pero incluye algunos nuevos. La clasificación está dividida en actores públicos o gubernamentales tales como: actores gubernamentales interestatales (AGI), actores gubernamentales no centrales (AGNC) y el Estado; y actores privados o no gubernamentales como los actores no gubernamentales interestatales (ANGI), los actores intraestatales no gubernamentales (AING) y el individuo (Barbé, 2010).
La presente investigación se focaliza en los actores gubernamentales no centrales (AGNC) también denominados actores subestatales o unidades subnacionales, los cuales "mantienen vínculos de diferentes tipo, promoviendo sus intereses a través de intercambios recíprocos, que generan efectos y entrelazan a diferentes sociedades y van institucionalizando novedosas formas de cooperación" (Colacrai y Zubelzú, 1998: 319 -320).

En cuanto a la Cooperación Descentralizada, la misma es entendida como una modalidad que consiste en acciones y vínculos basados en relaciones horizontales para el logro de beneficios mutuos desarrolladas entre administraciones subestatales de diferentes países tales como provincias, municipios, regiones, estados federados, entre otros. Dicho fenómeno adquiere un gran dinamismo y se repite en variadas regiones del mundo, no siendo el caso de Argentina un ejemplo aislado. Esto es aceptado por Colacrai y Zubelzú (1994) como una respuesta a los cambios que se producen en el sistema internacional tales como la globalización, la transnacionalidad, y una pérdida de la exclusividad del Estado.

Por otra parte, cuando un Estado adopta la forma federal, el poder se descentraliza políticamente bajo la figura de un Estado federal. Esta forma de gobierno establece la coexistencia de diversos niveles, sin estar subordinado uno al otro, ya que las relaciones están salvaguardadas constitucionalmente, evitando la subordinación tanto política como legal (Orlandi y Zelaznik, 1995). En Argentina, el Federalismo fue adoptado como la forma de gobierno e implica que el poder se encuentra descentralizado políticamente, por lo tanto coexisten tres niveles diferenciados pero con funciones concurrentes: el gobierno federal o nivel nacional, el nivel provincial que agrupa a los 23 gobiernos provinciales y el de la Ciudad Autónoma de Buenos Aires, y los gobiernos municipales.

Tras la Reforma de la Constitución Nacional Argentina en 1994, se estableció un nuevo marco legal, por el cual se fomentó la descentralización y se reconoció constitucionalmente la proyección internacional de las provincias (Art. Nro. $124 \mathrm{CN}$ ), impulsando el desarrollo de la gestión externa de los actores subestatales.

El traslado de competencias hacia las subunidades del gobierno central implicó el auge del fenómeno de la subestatalidad, caracterizado por una progresiva descentralización de políticas que eran propias del poder central, generando la asunción de prerrogativas propias de los gobiernos subnacionales (Russell, 2010). 
Por tal motivo, el Estado nacional argentino desarrolla acciones orientadas a difundir y acompañar la internacionalización de las provincias y la CD por medio del Ministerio de Relaciones Exteriores y Culto que dirige dichas acciones hacia los puntos focales localizados en las provincias argentinas. Se entiende por punto focal al área o referente provincial designado por el Poder Ejecutivo provincial cuya función es ser el nexo entre Cancillería y la provincia que representa, para el desarrollo de todo tipo de acciones de gestión externa.

Desde que las provincias encuentran reconocimiento sobre su accionar externo en la nueva Constitución Nacional de 1994, se comienza a debatir sobre la federalización de la política exterior (Colacrai y Zubelzú, 1994). Por tal motivo, pensar la articulación entre el Ministerio de Relaciones Exteriores y Culto de la Nación y las provincias de Argentina implica "la construcción de una relación coherente y eficaz entre ellas y la nación que evite la dispersión de las iniciativas aisladas y promueva el trabajo conjunto en la definición del interés nacional” (Iglesias, 2008: 8).

Teniendo en cuenta lo mencionado precedentemente a continuación se presentan tres apartados que pretenden desarrollar los contenidos para responder al objetivo propuesto: 1. La gestión externa de los actores subnacionales y la Cooperación Descentralizada, en el cual se describe el desarrollo y las características de la internacionalización de los actores subestatales; 2. El Ministerio de Relaciones Exteriores y Culto y la federalización de la Cooperación Internacional, apartado que aborda las funciones de las áreas de Cancillería relacionadas con la temática y los mecanismos de contactos con las provincias de Argentina; y 3. Articulación entre Cancillería y las Provincias de Argentina en materia de Cooperación Descentralizada, donde se desarrollan todos los programas y acciones de las áreas de Cancillería que fomentan la federalización de la Cooperación Descentralizada.

\section{La gestión externa de los actores subna- cionales y la Cooperación Descentralizada}

La internacionalización de los gobiernos locales y regionales es "un fenómeno prácticamente generalizado a nivel internacional que, pese a su aparente bajo perfil, estaría transformando de manera silenciosa algunos aspectos claves de la diplomacia clásica, y modificando la morfología de la política exterior" (Cornago Prieto, 2010: 108). Por tal motivo, el Estado-Nación desarrolla políticas que permiten delegar en los actores subnacionales algunas responsabilidades tomando decisiones que históricamente le correspondían al Estado como actor racional unificado.

Dentro del traslado de competencias es posible advertir acciones para la gestión de asuntos internacionales, desarrollando lo que se conoce como paradiplomacia. Considerada como la actividad internacional que, actuando a nivel global o transfronterizo, involucra a actores gubernamentales no centrales y gobiernos centrales o estaduales/provinciales/municipales extranjeros. Las motivaciones que impulsan la paradiplomacia pueden ser económicas, políticas o culturales (Keating, 2000).

Por su parte, Zubelzú (2006) sostiene que la gestión externa de las provincias resulta de un entramado de factores tales como: iniciativas a nivel subnacional por parte de estos actores, estímulos de tipo político y jurídico que han surgido a nivel nacional $y$, por último, factores que tienen que ver con los cambios sistémicos.

Existe una fuerte controversia terminológica respecto a este fenómeno. El primer trabajo de la mano de Duchacek y Soldatos utilizó el término paradiplomacia, aunque su mayor difusión fue alcanzada por Aldecoa y Keating, Lecours, Paquin, Mingus, Gelfenstein, Wolff y Zidaoui. Por otra parte, algunos autores han planteado alternativas al término tales como: diplomacia constitutiva - Kincaid - diplomacia multinivel - Hocking - diplomacia federativa - Shiavon, Rodriguez - diplomacia subestatal - Criekemans- acción exterior - Massart - o relaciones exteriores Hrbek, Michelmann (Cornago Prieto, 2010).

Por su parte, Colacrai y Zubelzú (1994) utilizan el término gestión externa o internacional para hacer referencia al accionar internacional de los actores subnacionales, por considerarse más adecuado al caso argentino.

A pesar de la diferencia terminológica y para comprender su alcance, es posible entender la acción externa de los entes subnacionales como: "La participación de los gobiernos no centrales en las Relaciones Internacionales, a través del establecimiento de contactos permanentes o ad hoc con entidades públicas o privadas extranjeras muy diversas, con el propósito de promover diversos aspectos socioeconómicos, culturales o medioambientales, así como cualquier otra dimensión de sus propias competencias constitucionales" (Cornago Prieto, 2010: 109).

Es posible identificar diversas variables sobre la gestión externa de los actores subestatales, que permite estable- 
cer la siguiente clasificación: 1. De acuerdo a las áreas temáticas abordadas en la gestión: es posible establecer distintas áreas de actividad internacional de los gobiernos estatales, tales como: la promoción de las exportaciones, la atracción extranjera directa, el apoyo a las comunidades en el exterior y la Cooperación Internacional; 2. De acuerdo a los actores intervinientes en la gestión externa: pudiendo ser públicos o privados como lo estable las categorías de Richard W. Mansbach, Yale H. Ferguson y Donald E. Lampert; y 3. De acuerdo a las áreas geográficas sobre las que se despliega el accionar externo: como regionales, fronterizas, o globales (Barreto, 2017).

Como se mencionó en el párrafo anterior la Cooperación Internacional, como parte de las acciones de gestión externa de los actores subestatales, es una herramienta entendida como "un proceso a través del cual las políticas seguidas por los gobiernos llegan a ser consideradas por los asociados como acciones que facilitan sus propios objetivos, como resultado de la coordinación de políticas" (Keohane, 1988: 88).

Existen diferentes modalidades de Cooperación Internacional por lo que es posible clasificarla de la siguiente manera (Alonso, 2009; Hourcade, 2011; Lechini, 2009):

De acuerdo al grado de desarrollo del donante: por un lado, la Cooperación Norte-Sur, tradicional o vertical, caracterizada por la ayuda de los países desarrollados a los países en desarrollo y, por otro lado, la Cooperación SurSur $u$ horizontal, cuya modalidad implica una cooperación entre países de similar desarrollo y entre estos y otro de menor desarrollo relativo. Asimismo es posible identificar a la denominada Cooperación triangular como una modalidad que articula a las dos anteriores, donde dos estados se unen para cooperar con un tercero.

De acuerdo a la temática abordada: cooperación humanitaria, científico-tecnológica, financiera, económica y técnica respondiendo a los temas en los que se va a cooperar.

De acuerdo a los actores que participan: cooperación multilateral (centralizada o descentralizada), en este caso el donante es una organización internacional, o se ven implicados varios estados en el proceso, o bilateral (centralizada, descentralizada o mixta) desarrollada entre dos estados. Asimismo existe una clasificación, de acuerdo con los actores, que permite diferenciar la Cooperación Centrali- $z a d a$, llevada a cabo por el Estado - Nación o sus órganos, de la Cooperación Descentralizada desarrollada por entidades subestatales de carácter público o privado.

La Cooperación Descentralizada es entendida como "una modalidad de cooperación liderada por los gobiernos locales, necesaria y complementaria a la cooperación bilateral y multilateral, cuyos objetivos e instrumentos permiten avanzar hacia formas de cooperación más simétricas, donde la reciprocidad y el aprendizaje mutuo se conviertan en ejes centrales de las relaciones entre gobiernos locales" (DGCIN, 2018) sin la necesaria intervención nacional. Resulta importante destacar que las referencias teóricas y conceptuales son actuales ya que el término CD apareció por primera vez en un documento oficial de la IV Convención de Lomé suscripto entre la Unión Europea y los países de África, Asia y Pacífico en el año 1989 (Grandas Estepa, 2011).

Esta modalidad de cooperación permite la participación directa de los gobiernos locales y genera el estímulo de procesos de descentralización impulsando la autonomía y la capacidad de gestión y decisión de los actores involucrados. Hourcade (2011) sostiene que, "Los principios rectores de la cooperación descentralizada son la horizontalidad, la reciprocidad y la simetría" (p.57).

\section{El Ministerio de Relaciones Exteriores y Culto, y la federalización de la Cooperación Internacional}

La política exterior de la República Argentina es conducida por el Poder Ejecutivo, que lleva adelante las negociaciones que se requieran para mantener la paz y las buenas relaciones con las demás naciones. El artículo Nro. 27 de la Constitución Nacional Argentina sostiene que: "El Gobierno federal está obligado a afianzar sus relaciones de paz y comercio con las potencias extranjeras por medio de tratados que estén en conformidad con los principios de derecho público establecidos en esta Constitución" (Art. Nro. $27 \mathrm{CN}$ ). Por tal motivo, y con el objetivo de poder gestionar las acciones pertinentes al desarrollo de la política internacional, se impulsó la creación del Ministerio de Relaciones Exteriores y Culto de la Nación ${ }^{1}$.

1. El Ministerio de Relaciones Exteriores cambió de denominación mediante la Ley 3727 del 1898 por el que se transforma en Ministerio de Relaciones Exteriores y Culto. Posteriormente en el año 1992 por la Ley 24190 pasó a ser llamado Ministerio de Relaciones Exteriores, Comercio Internacional y Culto. Finalmente por el Decreto 513 del año 2017 se le designó el nombre de Ministerio de Relaciones Exteriores y Culto, actualmente vigente. 
Para lograr la inserción internacional del Estado Argentino y, de acuerdo a las directivas que le imparte el Poder Ejecutivo Nacional, se le asigna a este Ministerio aquellas facultades que le permitan desempeñar y ejercer la política exterior. Conforme a las atribuciones de su competencia, dicho organismo determina sus objetivos y políticas.

En cuanto a la Cooperación, el Ministerio promueve y gestiona negociaciones en materia de Cooperación Internacional en distintos ámbitos, en coordinación con los demás ministerios y con los organismos nacionales que tengan competencia en dicha temática. Además promueve la elaboración de programas de cooperación en sus diversas modalidades. Consiguientemente, en materia de cooperación le corresponde a este organismo: "Entender, desde el punto de vista de la política exterior, en la negociación de la cooperación internacional en los ámbitos educativos, cultural, ambiental, económico, social, científico, técnico, tecnológico, nuclear, espacial, laboral y jurídico, en coordinación con los respectivos ministerios y con los demás organismos nacionales que tengan competencia en alguno de dichos ámbitos"(Art. 18 inc. 27 de Ley de Ministerios 22.520 - Texto ordenado por Decreto Nro. 438/92).

En Argentina, la Cooperación Internacional se gestiona principalmente desde el Ministerio de Relaciones Exteriores y Culto, federalizando la misma por medio de la $D i$ rección General de Cooperación Internacional (DGCIN), encargada de diagramar y gestionar proyectos bajo las diferentes modalidades de Cooperación Internacional, y la Dirección de Asuntos Federales y Electorales (DIRFE), área encargada de promover y coordinar acciones conjuntas con las entidades subnacionales, para lograr una mayor integración entre las diferentes realidades del territorio nacional (Decreto Nro. 174/ 18).

La Dirección General de Cooperación Internacional, actualmente dependiente de la Secretaría de Relaciones Exteriores, es el órgano encargado de formular diversas estrategias de cooperación, participar en las negociaciones pertinentes y coordinar las relaciones que existan entre Argentina y los distintos países del mundo respecto a esta temática. Según Cafiero (2008) "La Dirección General de Cooperación Internacional es el organismo responsable de la ejecución de la política exterior argentina en materia de cooperación técnica internacional” (p. 243).

Para el desarrollo de sus acciones la DGCIN cuenta con dos Redes Institucionales: 1. Soporte de politica exterior: apoyo de Representaciones Diplomáticas argentinas en todos los países del mundo, y 2. Soporte al interior del país en el país: basada en la contribución de una red de Puntos
Focales provinciales (Ministerio de Relaciones Exteriores y Culto - Dirección General de Cooperación Internacional, 2018).

Resulta importante destacar que, dicha Dirección General, lleva adelante acciones que posibiliten el desarrollo de proyectos bajo modalidades de cooperación diversas tales como: cooperación Sur- Sur, triangular, multilateral, bilateral, técnica o descentralizada.

Como parte de sus funciones, la DGCIN implementa diversos programas bajo la modalidad de la CD a fin de apoyar y fortalecer el desarrollo y su ejecución en los ámbitos provincial y local (Cafiero, 2008; Levi, 2011). Paralelamente, para lograr el fomento de la CD, la Dirección General difunde dichos programas y proyectos por medio de notas de prensa que promueven la participación de las Provincias o Municipios. Del análisis de notas relevadas se observa que la mayoría están relacionadas con las convocatorias que se encuentran abiertas y la promoción de actividades que visibilizan la difusión de las acciones de cooperación desarrolladas en algunas provincias, demostrando con ello el interés por acercar la Cancillería al interior del país.

Por otra parte, con el objetivo de federalizar sus proyectos, programas y acciones la DGCIN realiza reuniones anuales con los responsables de los puntos focales de las provincias de Argentina. Esta Red de Responsables de Cooperación Internacional está conformada por representantes de los Gobiernos provinciales y la Ciudad Autónoma de Buenos Aires, designados a ese efecto por los Gobernadores y Jefe de Gobierno de la Ciudad Autónoma.

Paralelamente al accionar de la DGCIN, la Dirección de Asuntos Federales y Electorales dependiente la Subsecretaría de Relaciones Institucionales y Diplomacia Pública promueve la vinculación entre el Estado Nacional, las Provincias y los Municipios para posibilitar el aprovechamiento de los recursos y servicios existentes a fin de promocionar el comercio exterior local, impulsar la integración e incrementar los vínculos fomentando la cooperación internacional.

La DIRFE asiste a las autoridades de los entes subestatales en la firma de Hermanamientos con los distintos municipios o provincias del exterior y coordina, con las Representaciones Diplomáticas y Consulares, las misiones oficiales al exterior de autoridades provinciales, municipales o regionales. Asimismo, dentro de las acciones propias de dicha área se destacan las capacitaciones técnicas sobre temas de interés de las provincias bajo los lineamientos 
de "Cancillería en el Interior" donde se dan a conocer las acciones para la promoción comercial, la planificación de misiones y los programas de cooperación Internacional disponibles por parte de Cancillería.

Resulta relevante destacar que: “[...] dado el número cada vez mayor de acciones externas emprendidas por las provincias argentinas, se hace necesario el asesoramiento y la colaboración de las Direcciones [...] que posibiliten un desempeño más eficiente, en un campo inexplorado por las provincias hasta hace no mucho tiempo atrás" (Colacrai y Zubelzú, 1994: 18). Del análisis de las Direcciones es posible observar que ambas desarrollan acciones muy puntuales con las entidades subestatales y no se evidencia un plan integrado para el abordaje conjunto de la temática.

Para concluir este apartado es relevante destacar que en el año 2008 se produjo una recategorización de los países de renta media, por parte del Banco Mundial y por el Comité de Ayuda al Desarrollo (CAD) de la Organización para la Cooperación y el Desarrollo Económicos (OCDE) tomando como parámetro la renta per cápita para determinar el grado de desarrollo de los países. Con esta iniciativa, la gran mayoría de los países latinoamericanos, que pertenecen al grupo de países de renta media, vieron limitado el acceso a los beneficios que ofrece la cooperación internacional con la retirada de importantes fuentes de financiamiento (Alonso, 2013). Por esta razón, considerando el nivel de ingreso de Argentina dicho país se encuentra en un nivel que no lo hace elegible para ser receptor de fondos de Ayuda Oficial al Desarrollo (Cafiero, 2008; Levi, 2011). Esta situación ha impulsado en Argentina la implementación de nuevas modalidades de Cooperación como por ejemplo la Cooperación Sur - Sur y la CD y el desarrollo de un renovado vínculo con el exterior donde los actores subnacionales son los protagonistas.

En este sentido, la DGCIN comenzó a desarrollar la CD como una alternativa, que propone como principios: "[...] establecer una base de igualdad entre los actores, la convergencia de los intereses mutuos, la complementariedad de las acciones y la participación activa de todos los implicados [...] a fin de apoyar y fortalecer su desarrollo y ejecución en los ámbitos provincial y local" (DGCIN, El Programa Federal, 2018) lo cual será analizado en el próximo apartado.

\section{Articulación entre Cancillería y las Pro- vincias de Argentina en materia de Coope- ración Descentralizada}

La mayoría de las acciones del Ministerio de Relaciones Exteriores y Culto en materia de CD apuntan a una colaboración técnica o a proyectos co-financiados por gobiernos locales que se complementen. Las iniciativas comprenden tareas de asistencia técnica, de formación y contactos con potenciales socios del exterior para la implementación de proyectos de desarrollo económico, social y cultural. También se ha dado un renovado impulso a la $\mathrm{CD}$, mediante la organización de foros e instancias de diálogo en múltiples niveles, habiéndose logrado, por ejemplo, acuerdos bilaterales con Francia que significaron la cofinanciación de 29 proyectos, 19 de los cuales son protagonizados por ciudades argentinas.

Existen diferentes tipos de alianzas entre gobiernos subnacionales identificadas por Cancillería para el desempeño de la CD: 1. Entre dos gobiernos de forma exclusiva, 2. Entre dos o más gobiernos subnacionales de diferentes países con agenda compartida y, 3. Entre dos o más gobiernos subnacionales de diferentes países, como ejes centrales de la alianza de cooperación, que trabajan a su vez coordinadamente con otros actores de sus comunidades.

De las publicaciones analizadas, se observa que las temáticas de la CD desarrollada por Argentina se focalizaron en las siguientes áreas: agricultura y agroalimentación, cultura, deporte, desarrollo urbano, economía y producción, educación, frontera, ambiente, salud y turismo.

Para el desarrollo de la CD, la DGCIN articula sus tareas junto al gobierno local argentino, el gobierno local extranjero y la Embajada Argentina en el país extranjero. En este proceso, resulta relevante destacar la vinculación entre la DGCIN y los gobiernos subnacionales argentinos, por medio de los Puntos Focales, siendo dicha Dirección la encargada de recibir las solicitudes y trasmitir las posibilidades de cooperación a los niveles provinciales y municipales, a fin de generar vínculos de cooperación impulsando la gestión externa. Posteriormente, la DGCIN remite las propuestas a la Red de embajadas argentinas en el exterior quienes de acuerdo al perfil del ente subestatal busca contrapartes para entablar una relación de cooperación fomentando, finalmente, los intercambios técnicos directos entre los gobiernos subnacionales sobre temática acordadas. 
Es por ello que, en cuanto al desempeño de la DGCIN, se observa que: "Su rol no es solamente de tipo instrumental, en tanto herramienta para la generación de acciones de cooperación, sino que también y principalmente es productora de políticas de Cooperación Internacional de la Cancillería, capaces de contribuir de manera sustantiva a los objetivos de la política exterior del país" (Levi, 2011: 27)

A continuación se desarrollan los principales programas y acciones implementadas en materia de CD y su federalización.

1. Programa Federal: Con el objetivo de avanzar hacia el fortalecimiento de un país federal y de optimizar la articulación entre las políticas establecidas en los diferentes niveles gubernamentales, en el año 2003 se creó el Programa Federal de Cooperación perteneciente a la DGCIN. Este Programa pretende favorecer un vínculo fluido entre los gobiernos provinciales y municipales, las universidades nacionales y las organizaciones no gubernamentales de Argentina para mejorar los trabajos conjuntos entre los diversos actores involucrados y difundir las oportunidades de Cooperación Técnica Internacional. A fin de implementar proyectos que favorezcan al desarrollo, la Dirección General, por medio del Programa, releva las necesidades y asiste a los gobiernos subnacionales en la identificación, búsqueda y gestión de la cooperación (Levi, 2011).

Las acciones desarrolladas en el marco del Programa Federal de Cancillería son implementadas a fin de articular las políticas nacionales, provinciales y municipales en materia de Cooperación Internacional. Para lograr esto, el Programa se propone: 1. Generar un vínculo de trabajo efectivo a través del relacionamiento con y entre los Responsables de Cooperación Internacional de las Provincias y la Ciudad de Buenos Aires, a través de la Red de Responsables de Cooperación, 2. Garantizar la adecuada distribución federal de la cooperación recibida, 3. Capacitar en cooperación técnica internacional y formulación de proyectos, 4. Articular con los Responsables de Cooperación Internacional de las provincias argentinas y la Ciudad de Buenos Aires, entre otros (DGCIN, Programa Federal de Cooperación, 2018).

La implementación del Programa implicó la creación de una Red de Responsables de Cooperación Internacional de las provincias y la Ciudad de Buenos Aires, constituida por funcionarios designados por los Señores Gobernadores y el Jefe de la Ciudad Autónoma de Buenos Aires.
Dentro de las acciones concretas que forman parte del Programa Federal se destacan los foros, encuentros, proyectos, convenios y programas específicos bilaterales con socios determinados. Es posible afirmar que el Programa se ha enfocado en fortalecer acciones tanto con socios del Norte como del Sur, con el objeto de garantizar una distribución federal de las oportunidades de cooperación promoviendo el desarrollo de las capacidades para potenciar el desarrollo local.

2. Programa COOPER.AR: La DGCIN, junto con la Secretaría de Asuntos Municipales de la Nación, hacia fines del 2014 presentó el Programa de Cooperación Internacional para Gobiernos y Municipios "COOPER.AR”, considerado la estrategia 2014-2015 para la federalización de la cooperación internacional.

El Programa se planteó los siguientes objetivos: 1. Coordinar las políticas en materia de Cooperación Internacional y las actividades de los actores de los diversos niveles del Estado en el exterior, 2. Contribuir con el fortalecimiento de los vínculos con las provincias y municipios en el área de Cooperación Internacional.

Para la implementación y desarrollo del Programa se planificó la realización de Jornadas de Cooperación en las provincias, capacitación en materia de formulación, monitoreo y evaluación de proyectos. La puesta en funcionamiento del Programa se basó en la realizaron reuniones con los puntos focales, con el objetivo de fortalecer el trabajo de la Red de Responsables de Cooperación de las provincias argentinas.

Como resultado en el año 2015 se publicó un catálogo de capacidades de Cooperación provincial denominado "Argentina Federal. Capacidades Provinciales para Cooperación Técnica. 120 experiencias para conocer y compartir", el cual compila las capacidades, fortalezas y aprendizajes provenientes de los gobiernos provinciales, que se ponen a disposición de la Cooperación Internacional desde su modalidad Subnacional o Descentralizada. El trabajo agrupa 120 capacidades de las 24 entidades subestatales que integran la República Argentina con el objetivo de potenciar y contribuir a una Cooperación cada día más federal.

\section{Acciones en materia de Cooperación Descentraliza-} da: las acciones de CD gestionadas por la DGCIN se enmarcan en el Programa Federal y pueden clasificarse en foros, encuentros, proyectos y convenios, siendo los destinatarios de dichas actividades las Provincias y Municipios de Argentina. 


\subsection{Foros y encuentros de CD:}

A. Encontrándonos con la Cooperación Descentralizada: programa desarrollado en el año 2005 por la DGCIN para el fomento de la CD en Argentina como herramienta para el fortalecimiento de las capacidades locales.

B. II Encuentro de Cooperación Descentralizada - Fomentando el Desarrollo Local: basado en el antecedente del Primer encuentro, en el año 2007 se desarrolló el II Encuentro destinado a Entidades Territoriales de la región. El mismo contó con una variedad de modalidades tales como exposiciones y disertaciones sobre diversas temáticas que fueron presentadas permitiendo dar a conocer las oportunidades de cooperación con el objetivo de generar alianzas estratégicas. Tras la participación de autoridades francesas, a partir de este Encuentro, y como fruto del trabajo conjunto con la Embajada Argentina en Francia y el Ministerio de Asuntos Exteriores de Francia con la DGCIN, se incrementó el compromiso por incentivar la CD entre ambos países y las entidades subnacionales.

\section{I Foro Franco-Argentino de Cooperación Descentraliza-} da: se realizó en el año 2008, en Buenos Aires y contó con la participación 16 gobiernos subnacionales de Francia y Representantes de Cooperación Internacional de las provincias y municipios argentinos. El objetivo primordial consistió en acercar a los actores subnacionales de Argentina con los gobiernos locales de Francia, a fin de que logren una identificación de temas de mutuo interés para que, en un segundo momento, se pudieran concretar actividades conjuntas en materia de cooperación descentralizada (Embajada Argentina en Francia, 2018). En el marco del Foro los representantes franceses viajaron al interior del país y visitaron a sus posibles contrapartes. En virtud de los resultados obtenidos a partir del I Foro se buscaron nuevos socios, se realizó el seguimiento de los vínculos que se habían comenzado y se lanzaron tres convocatorias para la presentación de proyectos.

D. II Encuentro Franco - argentino de Cooperación Descentralizada: la experiencia del primer foro propició la realización de un segundo Encuentro, que se llevó adelante en las ciudades de Biarritz y Pau del 12 al 14 de noviembre de 2012. El Encuentro permitió multiplicar las colaboraciones y fortalecer los intercambios entre los gobiernos, con la participación de más de 247 personas representando, entre otros, a 30 gobiernos locales argentinos y 44 franceses (Institut Français d'Argentine, 2018). En el marco del II Foro se realizaron visitas a terreno y, una vez concluido el evento, los representantes de los gobiernos subnaciona- les argentinos se trasladaron a localidades de sus socios franceses.

Uno de los principales resultados obtenidos fue una publicación del evento denominado " 2 " Encuentro Franco-Argentino de Cooperación Descentralizada” que releva las actividades, visitas, alianzas y acuerdos celebrados, para que sea un soporte impreso y digital que sirva de ejemplo para las vinculaciones de los entes subnacionales apuntando a considerar a los trabajos en conjunto, como una contribución para el desarrollo local. (Ministerio de Relaciones Exteriores y Culto, 20 Encuentro Franco- Argentino de Cooperación Descentralizada, 2012).

Es de destacar que desde el año 2008 se han incrementado la cantidad de vinculaciones con Francia contando hoy con alrededor de 30 alianzas entre gobiernos locales de ambos países, que llevan adelante trabajos conjuntos sobre 39 proyectos ejecutados en el marco de las seis convocatorias (DGCIN, 2018).

3.2. Proyectos y convenios bilaterales de Cooperación Descentralizada: una de las prioridades para la DGCIN, en cuanto a la $\mathrm{CD}$, se relaciona con el dictado de cursos de capacitación sobre el método el Ciclo de Manejo de Proyectos (PCM) para que los participantes puedan elaborar un Proyecto de Cooperación con consistencia lógica y así facilitar el proceso de solicitud de proyectos. En cuanto a las relaciones bilaterales, la Cancillería desarrolló los siguientes convenios y programas: Convenio de Cooperación con Cataluña, Programa de Cooperación con Japón, Cooperación con España, Programas de Cooperación con Alemania y Cooperación con Italia (Cooperación Argentina, 2018).

Resulta relevante destacar la referencia a la articulación tanto de políticas como de actores involucrados, anunciada por la Embajadora Julia Levi (2011) al afirmar que: "El propósito general es el del fortalecimiento del modelo de desarrollo autónomo del país [...], la articulación de políticas nacionales y provinciales con la cooperación internacional mediante el Programa Federal, y el fortaleciendo del multilateralismo, procurando que la DGCIN tenga una mayor inserción [...] promoviendo una articulación efectiva de la política de cooperación entre los distintos actores" (p. 28). 


\section{Conclusión}

A partir de los años setenta del siglo veinte se observa la pérdida de influencia del Estado - Nación y el surgimiento de corrientes transnacionalistas en el escenario internacional, por lo que es posible hallar nuevos actores que interactúan mutuamente de forma interdependiente. Esto ha impulsado una renovación en los análisis internacionales focalizando los estudios en dichos actores. De esta forma el escenario global se ha complejizado, dando como resultado el surgimiento de entidades subestatales que, formando parte de la esfera federal de un Estado, actúan a nivel global por medio de la cooperación como mecanismo para la gestión externa.

Tras la recategorización dada a los países de renta media, por parte del Banco Mundial y por el CAD de la OCDE, la gran mayoría de los países latinoamericanos, que pertenecen al grupo de países de renta media, vio limitado el acceso a los beneficios que ofrece la cooperación internacional (Cooperación Norte - Sur). Por esta razón, Argentina al transformarse en país de renta media, impulsó el desarrollo de nuevas modalidades de cooperación tales como la Cooperación Sur- Sur y Cooperación Descentralizada.

Este mecanismo de CD fue impulsado a partir de la Reforma de la Constitución Nacional de 1994 que, por el artículo $\mathrm{N}^{\circ} 124$, establece que las provincias puedan desarrollar acciones reconociendo la facultad de celebrar convenios internacionales, siempre que no sean incompatibles con la política exterior nacional. En el reconocimiento constitucional sobre la proyección internacional de las Provincias fue relevante el proceso de descentralización implementado de la década del '90 en Argentina.

La reforma de la Constitución del año 1994 propició el contexto normativo para la gestión externa de las provincias, demostrando con esto la concurrencia existente entre los actores subnacionales y el Estado federal.

Para lograr la inserción internacional del Estado Argentino y, de acuerdo a las directivas que le imparte el Poder Ejecutivo Nacional, se le asigna al Ministerio de Relaciones Exteriores y Culto aquellas facultades que le permitan desempeñar y ejercer la política exterior. Conforme a las atribuciones de su competencia, en la estructura de dicho Ministerio existen Áreas específicas para cumplir con los objetivos propuestos.

Considerando que uno de sus objetivos del Ministerio es la promoción y gestión de la Cooperación Internacional, se destaca la Dirección General de Cooperación Internacio- nal, área encargada de diagramar, difundir e implementar proyectos bajo las diferentes modalidades, y la Dirección de Asuntos Federales y Electorales que se encarga de la federalización de las acciones de Cancillería promoviendo acciones conjuntas con las entidades subnacionales para lograr una mayor integración entre las diferentes realidades del territorio nacional. En el ejercicio de las funciones de cada área, es posible observar que cada Dirección desempeña acciones de forma independiente, ya que dentro del organigrama no existe una interdependencia entre las áreas. Esto dificulta el trabajo coordinado, generando que las escasas acciones que realizan en forma conjunta no generen los resultados esperados.

El desarrollo de la CD como un nuevo mecanismo impulsado por la Cancillería intenta acompañar la solución de problemas cercanos y conocidos para los individuos y sociedades directamente involucrados teniendo en cuenta la multiplicación de actores y agentes intervinientes.

Resulta relevante destacar que la DGCIN es quien lleva adelante programas y acciones para el fomento de la CD, de acuerdo a las políticas fijadas por el gobierno nacional. Por tal motivo se ha implementado el Programa Federal de Cooperación desde el año 2003, apoyando los procesos de internacionalización de los gobiernos subnacionales. El Programa apunta a promover un vínculo fluido entre los actores subestatales públicos y privados de Argentina, para mejorar los trabajos conjuntos entre los actores involucrados, articulando las políticas nacionales, provinciales y municipales en materia de Cooperación, por medio de la difusión y el fomento de la cooperación técnica.

Uno de los ejes principales del Programa Federal es la articulación de acciones mediante una Red de Responsables de Cooperación Internacional de las provincias y de la Ciudad Autónoma de Buenos Aires, conformada por los puntos focales.

A partir de la sistematización de las acciones de CD de la DGCIN se observa que el Programa Federal se destaca como la mayor iniciativa de federalización, ya que el mismo está destinado a todas las provincias de Argentina, tiene una larga duración en el tiempo, estando actualmente vigente, aborda diversas temáticas y tiene un alto impacto local, ya que abarca una multiplicidad de actores intervinientes como de acciones.

Otra iniciativa para la difusión de la CD hacia los gobiernos subestatales lo constituyó el Programa de Cooperación Internacional para Gobiernos y Municipios "COO- 
PER.AR", creado por la DGCIN para ser aplicado en el periodo 2014-2015. La implementación del Programa implicó la realización de Jornadas de Cooperación en las provincias, capacitación en materia de formulación, monitoreo y evaluación de proyectos. Se destaca la Reunión Anual de Responsables Provinciales de Cooperación Internacional, que tuvo como objetivo compartir la estrategia de CD para fortalecer el trabajo de la Red de Responsables de Cooperación de las provincias argentinas. Asimismo, como resultado de dichas iniciativas, se publicó un catálogo de capacidades de Cooperación provincial y un documento de Cooperación Descentralizada de la Argentina.

En cuanto a otras acciones analizadas tales como foros, encuentros, asistencia técnica y proyectos, se destaca el II Encuentro Franco - argentino de Cooperación Descentralizada, como el proyecto que presenta mayores magnitudes, ya que generó una red de acciones bilaterales con una participación de múltiples actores y múltiples temáticas que se reflejan en la diversidad de proyectos presentados y ejecutados. Tuvo seis convocatorias que como resultado generaron 39 proyectos entre variadas provincias de Argentina y regiones de Francia, por lo que dicho proyecto se encuentra actualmente vigente.

Como herramienta para la difusión de las oportunidades en cooperación la DGCIN publica periódicamente una serie de informes, catálogos, balance de gestión, entre otros. Resulta relevante destacar que, en las notas de prensa se describen actividades que fueron llevadas a cabo en algunas provincias de Argentina, y se promocionan acciones de cooperación, demostrando con ello el interés por acercar la Cancillería al interior del país. A pesar de ello, se observa un reducido número de provincias visitadas, por lo que es posible afirmar que si bien el Ministerio de Relaciones Exteriores y Culto es de carácter Nacional, al tener sus oficinas en Buenos Aires y al no tener representaciones regionales, las actividades suelen quedar centralizadas en la capital.

Una acción de articulación concreta del Ministerio hacia las provincias consiste en la realización de la reunión anual con los responsables de los puntos focales de las provincias de Argentina. Como se dijo anteriormente, esta Red de Responsables de Cooperación Internacional está conformada por representantes de los Gobiernos provinciales y la Ciudad de Buenos Aires. En estos encuentros se difunden las oportunidades, proyectos, programas y acciones de la DGCIN en materia de Cooperación. Asimismo, se estimula a las provincias a trabajar en conjunto con Cancillería en el desarrollo de las gestiones de CD, como una herramienta que permite alcanzar el desarrollo local.
A partir de lo expuesto resulta relevante destacar que todas estas iniciativas de Cancillería hacia las provincias en materia de Cooperación refuerzan la idea de considerar una federalización de la política exterior. Colacrai y Zubelzú (1994) sostienen que en este proceso se potencian dos polos ya que se observa: "el poder central acercándose a las provincias y regiones, y éstas - o al menos algunas realimentando con audacia ese proceso" (p. 18).

En el actual escenario internacional, que continua transformándose, la globalización y el incremento de vinculaciones a todo nivel conforman un marco en el cual se diluye la posición exclusiva del Estado, abriendo paso a otros actores, que también proyectan su influencia o buscan satisfacer sus expectativas en un mundo cada vez más complejo. Por esta razón, la gestión externa como herramienta de política exterior seguirá profundizándose de la mano de la internacionalización de las entidades subestatales.

\section{Bibliografía}

ALONSO, José Antonio (2013) Cooperación con países de renta media: un enfoque basado en incentivos, AECID, [en línea], disponible en: https://bit.ly/2SkEOWr (Consulta: 26 de mayo de 2019)

BARALDI, Gildo, (2008), Aunando esfuerzos para la cooperación descentralizada: Articulación y Coordinación entre los actores (Conferencia del Observatorio de la Cooperación Descentralizada UE-AL), [en línea], disponible en: https://bit.ly/2JDgpsn (Consulta: 17 de marzo de 2019).

BARBE, Esther, (2010), Relaciones Internacionales, Tercera Edición, Editorial Technos, Madrid.

BARRETO, Maximiliano, (2017), La gestión internacional de las provincias argentinas. Una contribución al estudio de las modalidades subestatales de relacionamiento externo: el poder de vinculación mixto, Revista enfoques, volumen XXIX Nro.1, Editorial Universidad Adventista del Plata, [en línea], disponible en: https://bit.ly/2XU2XUX (Consulta: 18 de febrero de 2019)

CAFIERO, Ana (2008a), La Cooperación Descentralizada en la Argentina, Revista Estudios de casos, [en línea], disponible en: https://bit. ly/2JA5nnE (Consulta: 26 de enero de 2019).

CAFIERO, Ana (2008b), "Escenarios de la Cooperación Internacional en la Argentina" en IGLESIAS, Eduardo, Las provincias argentinas en el escenario internacional Desafíos y obstáculos de un sistema federal, CARI - PNUD, Buenos Aires.

CALVENTO, Mariana (2015), La Política Internacional Subnacional: una propuesta para el abordaje del accionar contemporáneo de Argentina, Desafíos, Bogotá

COLACRAI de TREVISAN, Miryam y ZUBELZÚ de BACIGALUPO, Graciela, (1998), El creciente protagonismo externo de las provincias argentinas, CERIR, La política exterior argentina 1994/1997, Rosario 
COLACRAI, Miryam y ZUBELZÚ, Graciela, (1994), Las provincias y sus relaciones externas. ¿Federalización de la Política Exterior o protagonismo provincial en las relaciones internacionales?, CERIR, Cuadernos de Política Exterior $N^{\circ}$ 52, Rosario.

COLACRAI, Miryam, (2011), Cooperación al desarrollo: diferentes lecturas para reflexionar acerca de sus motivaciones y objetivos, ponencia presentada en SAAP, Córdoba, julio.

Constitución de la Nación Argentina, versión digital disponible en: shorturl.at/ixBCK

CORNAGO PRIETO, Noé, (2010), "La descentralización como elemento de innovación diplomática”, en MAIRA, Luis (Ed.), La Política Internacional Subnacional en América Latina, Libros del Zorzal, Buenos Aires.

Decisión Administrativa No 308, PEN, 2018, versión digital disponible en: https://bit.ly/2LYe2Sy

Decreto No 174, PEN, 2018, versión digital disponible en: https://bit. ly/2uYP6AC

DGCIN (2014), $2^{\circ}$ Encuentro Franco-Argentino de Cooperación Descentralizada., [en línea], disponible en: https://bit.ly/2JDmdCe (Consulta: 18 de enero de 2019).

DGCIN (2015). Argentina Federal. Capacidades Provinciales para Cooperación Técnica. 120 experiencias para conocer y compartir, [en línea], disponible en: https://bit.ly/2eG5Bdq (Consulta: 18 de enero de 2019).

GARCIA SEGURA, Caterina, (1996), La actividad exterior de las entidades políticas subestatales, en Revista de Estudios Políticos (Nueva Época), $N^{\circ}$ 91, enero-marzo.

GRANDAS ESTEPA, Denisse, (2011), "La cooperación descentralizada como un nuevo modelo en cooperación para el desarrollo", [en línea], en Revista Análisis Internacional, disponible en: https://bit. ly/2IoAGDm (Consulta: 25 de enero de 2019).

HERNÁNDEZ, Antonio (2005), "La descentralización del poder en el Estado Argentino”, en HERNÁNDEZ Antonio, La descentralización del poder en el estado contemporáneo, Asociación Argentina de Derecho Constitucional, Buenos Aires.

HOURCADE, Odile, (2011), "Concepto y alcance de la cooperación descentralizada, en Revista Trabajos de Investigación en Paradiplomacia”, Año 1, Número 1, diciembre, Buenos Aires, [en línea], disponible en: https://bit.ly/30DtS8W (Consulta: 05 de febrero de 2019).

IGLESIAS, Eduardo, (2008), Las provincias argentinas en el escenario internacional. Desafíos y obstáculos de un sistema federal, 1ra. Ed., Buenos Aires, PNUD.

KEATING, Michael, (2000), "Regiones y asuntos internacionales: motivos, oportunidades y estrategias", en ALDECOA, Francisco y KEATING, Michael, (Eds), Paradiplomacia: las relaciones internacionales de las regiones, Marcial Pons, Ediciones Jurídicas y Sociales SA, Madrid.

KEOHANE, Robert y NYE, Joseph, (1988), Poder e interdependencia: la política mundial en transición, GEL, Buenos Aires.

KEOHANE, Robert, (1988), Después de la Hegemonía. Cooperación y discordia en la política mundial, GEL, Buenos Aires.

LECHINI, Gladys, (2009), "Argentina y Brasil en la Cooperación SurSur”, en LECHINI, Gladys, KLAGSBRUNN, Victor y GONCALVES
Williams (Org./Comp.), Argentina y Brasil. Venciendo preconceptos. Las variadas aristas de una concepción estratégica, Editora Revan, Brasil.

LEVI, Julia, (2011), La Argentina y la nueva arquitectura de la cooperación internacional, Revista Española de Desarrollo y Cooperación, Instituto Universitario de Desarrollo y Cooperación, Madrid.

Ley de Ministerios 22.520, 12 de marzo de 1992, versión digital disponible en: https://bit.ly/2DC0Lw8

MAIRA, Luis (editor), (2010), La Política Internacional Subnacional en América Latina, Libros del Zorzal, Buenos Aires.

ORLANDI, Hipólito y ZELANIK, Javier (1995) "El gobierno" en Pinto, Julio (compilador). "Manual de Introducción a la Ciencia Política". Eudeba, Buenos Aires

RUSSELL, Roberto, (2010), “El Estado Nación y los actores gubernamentales no centrales: una relación complementaria”, en MAIRA, Luis (Ed.), La Política Internacional Subnacional en América Latina, Libros del Zorzal, Buenos Aires.

SALOMON GONZALEZ, Mónica, (2001-2002), La teoría de las relaciones internacionales en los albores del siglo XXI: diálogo, disidencia y aproximaciones, Revista CIDOB d'Afers Internacionals, $\mathrm{N}^{\circ} 56$.

SALVIOLO, Marcelo, (2005), Provincias y convenios internacionales: una propuesta desde el Federalismo de Concertación, Nuevo Hacer GEL, Buenos Aires.

ZUBELZÚ, Graciela (2006), La acción Internacional de las provincias argentinas. Reflexiones generales y análisis de algunos perfiles de gestión. Centro de Estudios Avanzados, Universidad Nacional de Córdoba.

ZUBELZÚ, Graciela (2008a), "Los gobiernos subnacionales en el escenario internacional: concepto, variantes y alcance. Un marco de análisis para las acciones de las provincias argentinas", en IGLESIAS, Eduardo, Las provincias argentinas en el escenario internacional. Desafíos y obstáculos de un sistema federal, 1ra. Ed, PNUD Buenos Aires.

ZUBELZÚ, Graciela (2008b), "El diseño institucional y los perfiles de gestión externa de las provincias argentinas", en IGLESIAS, Eduardo, Las provincias argentinas en el escenario internacional. Desafíos y obstáculos de un sistema federal, 1ra. Ed., PNUD, Buenos Aires.

Páginas de internet consultadas

Comité de Ayuda al Desarrollo: www.oecd.org/dac Cooperación Argentina: www.cooperacionarg.gob.ar Embajada Argentina en Francia: www.efran.cancilleria.gob.ar Información Legislativa y Gubernamental: www.infoleg.gob.ar InstitutFrançaisd'Argentine: www.ifargentine.com.ar Ministerio de Relaciones Exteriores y Culto de la Nación: www.mrecic.gov.ar

Organización para la Cooperación y el Desarrollo Económico: www.oecd.org

Recibido 3 de mayo 2019- Aceptado 18 de mayo 2019 